Intestinal obstruction is the presenting symptom in $40 \%$ of patients with colorectal cancer. Emergency operative therapy is often suboptimal and associated with significant morbidity and mortality $[1-3]$. The use of endoluminal stents as a temporary measure to relieve the obstruction caused by these lesions has been reported. Successful stenting allows time to prepare the bowel and work-up the patient prior to definitive surgery [4, 5]. Large-scale randomized trials to define the role of endoluminal stenting are awaited. We would like to report an uncommon site of perforation associated with the procedure.

A 63-year-old man presented with a 1week history of abdominal distension and absolute constipation. An abdominal radiograph revealed large-bowel intestinal obstruction. An urgent water-soluble contrast enema showed complete obstruction at the sigmoid colon. Stent insertion was attempted using a dual-channel endoscope (GIF2T240; Olympus, Tokyo, Japan). The lumen was cannulated and then a Zebra guide wire was passed. A $22 \mathrm{~mm} \times 60 \mathrm{~mm}$ Enteral Wallstent (Boston Scientific Microvasive, Minnesota, USA) was railroaded by means of the guide wire to the desired position, with the aid of the endoscope and fluoroscopy. There were some difficulties in negotiat-

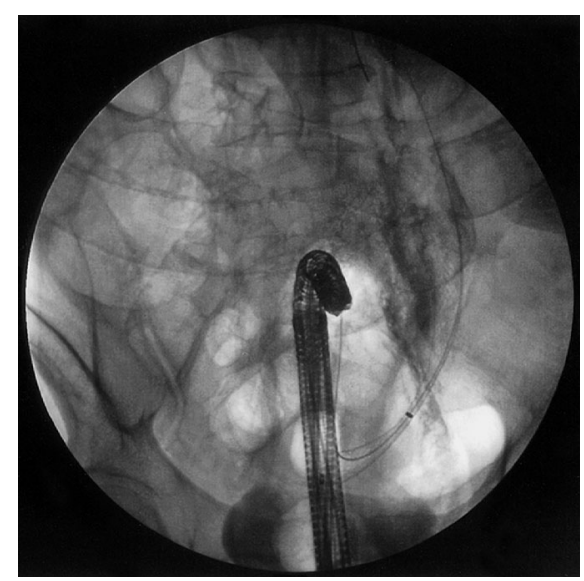

Figure 1 The stent fully deployed in the colon under fluoroscopic guidance.

\title{
Mesenteric Perforation of an Obstructing Sigmoid Colon Tumor after Endoluminal Stent Insertion
}

ing the stent through the stricture, but the stent could be seen on fluoroscopy to have expanded successfully (Figure $\mathbf{1}$ ).

There was no passage of gas or stool after stent deployment. After 1 hour, subcutaneous emphysema was noted. The patient immediately underwent a laparotomy to deal with an obstructing sigmoid colon tumor (T4N1M0) with the stent located in the sigmoid mesentery (Figure 2). A Hartmann's procedure was performed, and the patient made an uneventful recovery.

While endoluminal stenting is an attractive temporary minimally invasive method for treating an obstructing sigmoid tumor, its potential complications cannot be overemphasized. In this patient, we postulated that the tumor was perforated at the mesenteric side by the guide wire that was passed via the cannula. The false tract was then dilated and the stent inserted into the sigmoid mesentery. This explained why we had difficulty in negotiating the stent through the tumor. This case illustrated again the importance of continuous monitoring of patients after endoscopic procedures and of early surgical intervention in cases where complications arise.

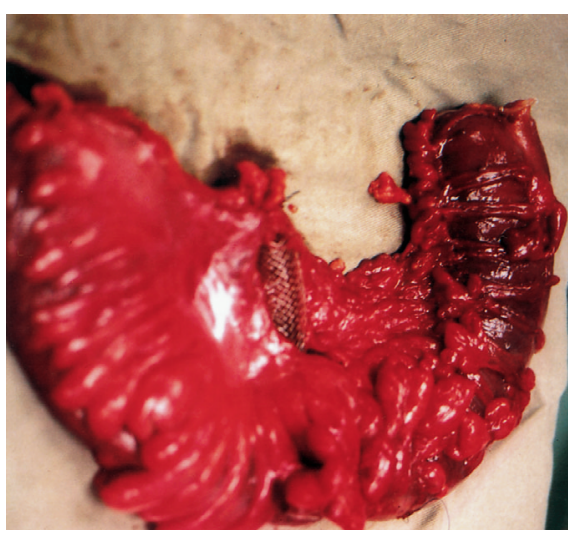

Figure 2 Intraoperative finding: the stent located in the sigmoid colon mesentery.

\author{
R. I. Sarmiento, D. W. H. Lee, \\ S. K. H. Wong, A. C. W. Chan, \\ S. C.S. Chung \\ Department of Surgery,
}

Prince of Wales Hospital,

The Chinese University of Hong Kong,

Hong Kong

\section{References}

${ }^{1}$ Arnell T, Stamos MJ, Takahashi P et al. Colonic stents in colorectal obstruction.

Am Surg 1998; 64: 986-988

${ }^{2}$ Lo SK. Metallic stenting for colorectal obstruction. Gastrointest Endosc Clin N Am 1999; 9: 459 - 477

${ }^{3}$ Saida Y, Sumiyana Y, Nagao J, Takase M. Stent endoprosthesis for obstructing colorectal cancers. Dis Colon Rectum 1996; 39: 552-555

${ }^{4}$ Carneros JA, Senent MC, Vaquero J et al. The endoscopic placement of self-expanding metal prosthesis as palliative treatment in colorectal stenosis of tumor origin. Gastroenterol Hepatol 1999; 22: 167 - 170

${ }^{5}$ Boorman P, Soonawalla Z, Sathananthan $\mathrm{N}$ et al. Endoluminal stenting of obstructed colorectal tumors. Ann R Coll Sur Engl 1999; 81: 251 - 254

\section{Corresponding Author}

\section{S. C. S. Chung, M.D.}

Prince of Wales Hospital The Chinese University of Hong Kong Shatin, N.T.

Hong Kong SAR

China

Fax: $\quad+852-2637-7974$

E-mail: sydneychung@cuhk.edu.hk 